\title{
AUTO-ESTIMA EM PACIENTES COM CARCINOMAS DE PELE
}

\section{SELF-ESTEEM OUTCOMES AMONG NON MELANOMA SKIN CANCER PATIENTS}

\author{
Marcelo Prado de Carvalho'; Renato Santos de Oliveira Filho, TCBC-SP2; \\ Heitor Carvalho Gomes ${ }^{2}$; Daniela Francescato Veiga ${ }^{3}$; Yara Juliano ${ }^{4}$; Lydia Masako Ferreira ${ }^{5}$
}

\begin{abstract}
RESUMO: Objetivo: Avaliar a auto-estima de pacientes com carcinomas de pele da face e do pescoço. Métodos: Foram estudados 100 pacientes, com idades entre 30 e 90 anos (mediana $=55$ anos), sendo 44 eram do gênero masculino e 56 do gênero feminino. O Grupo Estudo foi composto por 50 pacientes portadores de carcinomas de pele na face e no pescoço, com tamanho igual ou maior a $10 \mathrm{~mm}$ no seu maior eixo e com diagnóstico confirmado por biópsia prévia. O Grupo Controle consistiu de 50 indivíduos da população geral. Para avaliação da auto-estima, utilizou-se a Escala de Auto-estima de Rosenberg / UNIFESP-EPM. A análise estatística utilizou com o teste de Mann-Whitney e a Análise de Variância de Kruskal Wallis. Resultados: Os pacientes portadores de câncer de pele e os da população geral não apresentaram diferenças significantes em relação à idade ( $\mathrm{p}=0,331)$, gênero e escolaridade. O tamanho médio do tumor foi $20,9 \pm 2 \mathrm{~mm}$. Os pacientes com câncer de pele apresentaram auto-estima significantemente mais baixa, com escore médio de 6,84 e 3,90 para o Grupo Controle ( $\mathrm{p}=0,002)$. Conclusão: Os pacientes com câncer de pele na face e no pescoço apresentaram auto-estima menor do que os pacientes sem câncer (Rev. Col. Bras. Cir. 2007; 34(6): 361-366).
\end{abstract}

Descritores: Neoplasias cutâneas; Carcinoma basocelular; Carcinoma de células escamosas; Neoplasias de cabeça e pescoço; Qualidade de vida; Auto-imagem.

\section{INTRODUÇÃO}

As neoplasias malignas da pele são os tumores mais comuns adquiridos pelo ser humano no curso de sua vida. $\mathrm{O}$ carcinoma basocelular (CBC) e o carcinoma espinocelular (CEC) são os tumores mais freqüentes e, por isso, são de muito interesse à saúde pública em muitos países. Epidemiologicamente, estima-se um risco de acometimento por carcinomas de pele de 28 a $33 \%$ para o CBC e de 7 a $11 \%$ para o CEC. A incidência de carcinomas de pele supera a incidência de cânceres do pulmão, mama, cólon, reto, próstata, bexiga e todos os linfomas combinados ${ }^{1}$.

Os cânceres de pele localizados na face e no pescoço podem resultar em morbidade muito significativa por causa do valor funcional e cosmético que estas regiões apresentam. Há muito tempo a pele possui grande importância do ponto de vista psicológico, influenciando de maneira bastante peculiar à vida emocional do homen. Pode haver conseqüências adversas, que incluem distúrbios psíquicos e psicossociais, após a ocorrência de deformidades causadas por este tipo de câncer² .

Nos últimos 30 anos, diversos instrumentos para avaliação da qualidade de vida têm sido desenvolvidos, geralmente para comparar resultados de tratamentos (a maioria aplicada em estudos para o câncer ou doenças crônicas), no contexto de estudos clínicos ${ }^{3}$. A Qualidade de Vida é descrita como sendo formada pela percepção do indivíduo sobre o seu estado de saúde em relação aos aspectos social, físico, psicológico, econômico e espiritual ${ }^{4}$. E a auto-estima, que é um componente da qualidade de vida, é definida como sendo o sentimento, o apreço e a consideração que uma pessoa sente por si própria, ou seja, o quanto ela gosta de si, como ela se vê e o que pensa sobre ela mesma 5 .

A escala de auto-estima de Rosenberg/UNIFESPEPM é um instrumento específico de avaliação da qualidade de vida, que aborda este importante aspecto da vida humana, a auto-estima ${ }^{5}$.

Com o objetivo avaliar a auto-estima de pacientes com carcinomas de pele da face e do pescoço realizou-se o presente estudo.

\section{MÉTODOS}

Realizou-se um estudo, do tipo analítico transversal, previamente aprovado pela Comissão de Ética em Pesquisa, em que participaram 100 indivíduos atendidos nos Ambulatórios do Hospital das Clínicas Samuel Libânio da Universidade do Vale do Sapucaí, de Pouso Alegre, Minas Gerais, no perío-

1. Pós Graduando do Mestrado Interinstitucional, MINTER UNIFESP-UNIVÁS.

2 .Professor Orientador do Programa de Pós-Graduação em Cirurgia Plástica da UNIFESP e Professor Doutor Colaborador da Disciplina de Cirurgia Plástica -UNIFESP.

3. Professora Doutora Coordenadora Operacional do MINTER UNIFESP-UNIVÁS.

4. Professora Doutora Colaboradora dos Departamentos de Bioestatística da UNIVÁS e UNIFESP.

5. Professora Titular; Chefe da Disciplina de Cirurgia Plástica e Coordenadora do Programa de Pós-Graduação em Cirurgia Plástica da UNIFESP.

Recebido em 05/04/2007

Aceito para publicação em 04/06/2007

Conflito de interesses: nenhum

Fonte de financiamento: nenhuma

Trabalho realizado nos Ambulatórios do Hospital das Clínicas Samuel Libânio da Universidade do Vale do Sapucaí, de Pouso Alegre - Minas Gerais. 
do de maio de 2005 a janeiro de 2006. Selecionou-se, consecutivamente, 50 pacientes portadores de carcinomas da pele da face e do pescoço, com idade entre 30 e 90 anos, nos ambulatórios de Cirurgia Plástica e de Dermatologia que, após consentimento informado, foram avaliados em um estudo sóciodemográfico e por uma Escala de Auto-estima. Um grupo de 50 indivíduos, com distribuição semelhante quanto à faixa etária, gênero e escolaridade, não portadores de tumores cutâneos, formou o grupo controle.

Foram selecionados para o Grupo Estudo indivíduos com carcinomas do tipo basocelular ou espinocelular, com diagnóstico confirmado por biópsia incisional prévia, com diâmetro igual ou maior que $10 \mathrm{~mm}$ no seu maior eixo. Foram excluídos aqueles com diagnóstico diferente de carcinoma cutâneo à biópsia ou com presença concomitante de qualquer outro tipo de neoplasia. Também foram excluídos os portadores de doenças sistêmicas não controladas, doenças de caráter severo, crônico ou recidivante.

A Escala de Auto-estima Rosenberg/UNIFESPEPM foi aplicada através de entrevista, pelo autor, realizada em sala do ambulatório médico. A todos os pacientes incluídos neste estudo foi garantido o tratamento da doença na própria Instituição. Esta escala é um instrumento específico de avaliação deste importante aspecto da Qualidade de Vida (auto-estima), traduzido, adaptado e validado ao contexto cultural brasileiro. É composto por dez questões: 1) De uma forma geral (apesar de tudo), estou satisfeito(a) comigo mesmo(a); 2) Às vezes, eu acho que eu não sirvo para nada (desqualificado ou inferior em relação aos outros); 3) Eu sinto que eu tenho um tanto (um número) de boas qualidades; 4) Eu sou capaz de fazer coisas tão bem quanto a maioria das outras pessoas (desde que me ensinadas); 5) Não sinto satisfação nas coisas que realizei. Eu sinto que não tenho muito do que me orgulhar; 6) Às vezes, eu realmente me sinto inútil (incapaz de fazer as coisas); 7) Eu sinto que sou uma pessoa de valor, pelo menos num plano igual (num mesmo nível) às outras pessoas; 8) Não me dou o devido valor. Gostaria de ter mais respeito por mim mesmo(a); 9) Quase sempre eu estou inclinado(a) a achar que sou um(a) fracassado(a); 10) Eu tenho uma atitude positiva (pensamentos, atos e sentimentos positivos) em relação a mim mesmo ${ }^{5}$. A Escala apresenta quatro opções de resposta: concordo plenamente; concordo; discordo; discordo plenamente ${ }^{4}$. Os resultados foram computados após a soma dos valores encontrados em um gabarito. Neste instrumento quanto maior o escore, menor a autoestima do indivíduo.

Para a análise dos resultados foram aplicados o Teste do Qui quadrado para estudar associações entre fototipos de pele e escolaridade dos indivíduos em relação aos grupos com e sem câncer de pele ${ }^{6}$, e o Teste de MannWhitney com o objetivo de comparar os grupos etários menor ou igual a 55 anos e maior ou igual a 56 anos em relação à Escala de Auto-estima ${ }^{5}$. O mesmo teste foi aplicado para comparar os grupos Controle e Estudo. A Análise de variância de Kruskal Wallis, com a finalidade de comparar as regiões da face e o tamanho do tumor em relação aos resultados do questionário ${ }^{6,7}$.
A formulação da hipótese alternativa $\left(\mathrm{H}_{1}\right)$ foi monocaudal, isto é, para todas as questões do questionário a hipótese foi a de observar valores maiores para o Grupo Estudo na Escala de Auto-estima. Fixou-se em 0,05 ou $5 \%$ o nível de rejeição da hipótese $\mathrm{H}_{0}$, assinalando com um asterisco os valores significantes.

\section{RESULTADOS}

Os pacientes portadores de carcinomas de pele da face e do pescoço apresentaram média etária de 57,9 \pm 13,5 anos e os do Grupo Controle apresentaram idade média de 55,1 1 12,5 anos, não havendo diferença estatisticamente significante entre os grupos. A Tabela 1 apresenta as características sóciodemográficas dos dois grupos.

Os maiores escores da Escala de Auto-estima de Rosenberg/ UNIFESP-EPM foram observados no grupo que envolvia pacientes com câncer de pele (média $=6,8$; mediana $=$ 7,0 ), representando uma baixa auto-estima em relação ao grupo sem câncer (controle) (Tabela 2).

Realizou-se, também, após análise estatística e com a mediana calculada, uma divisão em duas faixas etárias, a saber, pacientes mais jovens, com idade igual ou inferior a 55 anos e outro igual ou superior a 56 anos, comparando-se entre si e entre os grupos Estudo e Controle (Figura 1). Foram encontrados resultados significantes na parcela mais jovem do Grupo Estudo que apresentou menor auto-estima $(\mathrm{p}=0,002)$ (Tabela 3 ).

A maior freqüência de tumores de pele na cabeça e no pescoço ocorreu em regiões de grande exposição como as regiões nasal, oral, orbital, bochecha, zigomática, cervical e têmporo - frontal (Tabela 4). A partir desta subdivisão da face, realizou-se verificação estatística destas regiões entre si, com os escores obtidos, não sendo identificado diferença significante $(\mathrm{p}=0,322)$.

A maior incidência de carcinomas de pele ocorreu nos indivíduos de pele clara, ou seja, aqueles com pele branca e sensível, nas classes II e III da classificação de Fitzpatrick ${ }^{6}$ (Tabela 5).

Com o objetivo de excluir influências do tamanho da neoplasia sobre a auto-estima do indivíduo, foram estabelecidos quatro grupos, sendo o Grupo 1 com tumores que variavam de 10 a 19 milímetros, o Grupo 11 de 20 a 29 mm, o Grupo 111 de 30 a 39 mm e o Grupo IV de 40 a $52 \mathrm{~mm}$. Comparando-se os grupos pela Análise de Variância observou-se não haver relevância estatística para o tamanho da lesão no resultado deste estudo $(\mathrm{p}=0,126)$.

\section{DISCUSSÃO}

Em estatísticas norte-americanas, um em cada cinco indivíduos de pele clara, em algum momento de suas vidas, adquirirá um tipo de câncer cutâneo ${ }^{1}$. O CBC e o CEC são os tumores mais freqüentes e interessam muito à saúde pública nos diferentes países. Estimativas recentes predizem que, só nos Estados Unidos, ocorrem 1,3 milhões de novos casos todos os anos, esperando-se que este valor dobre nos próximos $30 \operatorname{anos}^{1,3,8,9}$. Para esses americanos de pele clara, estima- 
Tabela 1 - Indivíduos com câncer e sem câncer de pele, segundo características sócio-demográficas (n=100).

\begin{tabular}{|c|c|c|c|c|}
\hline \multirow{2}{*}{$\begin{array}{l}\text { Características } \\
\text { Idade, anos, média }\end{array}$} & \multicolumn{2}{|c|}{ Pacientes com câncer de pele $n=50$} & \multicolumn{2}{|c|}{ Pacientes sem câncer de pele $n=50$} \\
\hline & \multicolumn{2}{|c|}{57,86} & \multicolumn{2}{|c|}{55,14} \\
\hline Gênero Masculino & 26 & $(52,0)$ & 18 & $(36,0)$ \\
\hline Gênero Feminino & 24 & $(48,0)$ & 32 & $(64,0)$ \\
\hline \multicolumn{5}{|l|}{ Estado marital-n (\%) } \\
\hline Com companheiro & 36 & $(72,0)$ & 36 & $(72,0)$ \\
\hline Sem companheiro & 14 & $(28,0)$ & 14 & $(28,0)$ \\
\hline \multicolumn{5}{|l|}{ Fototipos de Fitzpatrick (\%) } \\
\hline $\mathrm{I}$ & 2 & $(4,0)$ & 1 & $(2,0)$ \\
\hline II & 32 & $(64,0)$ & 27 & $(54,0)$ \\
\hline III & 11 & $(22,0)$ & 13 & $(26,0)$ \\
\hline IV & 5 & $(10,0)$ & 3 & $(6,0)$ \\
\hline $\mathrm{V}$ & 0 & & 5 & $(10,0)$ \\
\hline VI & 0 & & 1 & $(2,0)$ \\
\hline \multicolumn{5}{|l|}{ Comorbidades } \\
\hline Hipertensão arterial & 21 & $(42,0)$ & 18 & $(36,0)$ \\
\hline Diabetes & 6 & $(12,0)$ & 5 & $(10,0)$ \\
\hline \multicolumn{5}{|l|}{ Escolaridade-n(\%) } \\
\hline Sem instrução & 8 & $(16,0)$ & 2 & $(4,0)$ \\
\hline Fundamental & 37 & $(74,0)$ & 32 & $(64,0)$ \\
\hline médio & 5 & $(10,0)$ & 11 & $(22,0)$ \\
\hline superior & 0 & $(0,0)$ & 5 & $(10,0)$ \\
\hline \multicolumn{5}{|l|}{ Remuneração } \\
\hline Atividade remunerada & 30 & $(60,0)$ & 33 & $(66,0)$ \\
\hline Sem ativ. Remunerada & 20 & $(40,0)$ & 17 & $(34,0)$ \\
\hline \multicolumn{5}{|l|}{ Ocupação } \\
\hline Ativ. no meio rural & 14 & $(28,0)$ & 10 & $(20,0)$ \\
\hline Const. Civil/ exposição sol & 5 & $(10,0)$ & 5 & $(10,0)$ \\
\hline Trab. Recinto fechado & 12 & $(24,0)$ & 16 & $(32,0)$ \\
\hline Do lar / aposentado & 19 & $(38,0)$ & 19 & $(38,0)$ \\
\hline
\end{tabular}

se um risco de acometimento de 28 a $33 \%$ para o CBC e de 7 a $11 \%$ para o $\mathrm{CEC}^{1}$. Os cânceres de pele estão ficando cada vez mais comuns e sua localização em regiões da face pode levar a sérias deformidades ${ }^{10}$.

Segundo o Instituto Nacional do Câncer do Brasil (INCA), o número de casos novos de carcinomas de pele estimados para o Brasil em 2006 foi de 55.480 casos em homens e de 61.160 casos em mulheres ${ }^{11}$. Apesar de continuar sendo a neoplasia mais incidente em nosso país, em ambos os gêneros, considera-se que estes dados estejam subestimados pelo fato de que muitas lesões suspeitas são retiradas sem diagnóstico. Conseqüentemente, as estimativas das taxas de incidência e de números esperados de novos casos são consideradas mínimas ${ }^{11}$.

Os integrantes deste estudo eram pertencentes em sua maioria, a estratos sociais menos favorecidos e de baixa escolaridade (Tabela 1). Uma parcela dos indivíduos nos dois grupos era oriunda de cidades de pequeno porte e possuíam fototipos de pele semelhantes (diferença não significante), coincidindo com as referências na literatura sobre a exposição

Tabela 2 - Indivíduos com câncer de pele (grupo estudo) e sem câncer de pele (grupo controle) segundo a idade e os escores da Escala de Auto-estima Rosenberg/UNIFESP-EPM - Resultado do teste de Mann-Whitney (z).

\begin{tabular}{ccccc}
\hline & & Idade & & \multicolumn{2}{c}{ Auto-estima } \\
& Estudo & Controle & Estudo & Controle \\
\hline $\mathrm{X}$ & 57,86 & 55,14 & 6,84 & 3,90 \\
$\mathrm{Mi}$ & 55,5 & 55,0 & 7,00 & 3,50 \\
& $\mathrm{Zcalc}=0,97$ & $(\mathrm{p}=0,331)$ & Zcalc=3,11 & $(\mathrm{p}=0,002)$ \\
\hline
\end{tabular}


Tabela 3 - Indivíduos com câncer de pele (grupo estudo) ou sem câncer de pele (grupo controle) segundo os escores da Escala de Auto-estima de Rosenberg / UNIFESP-EPM.

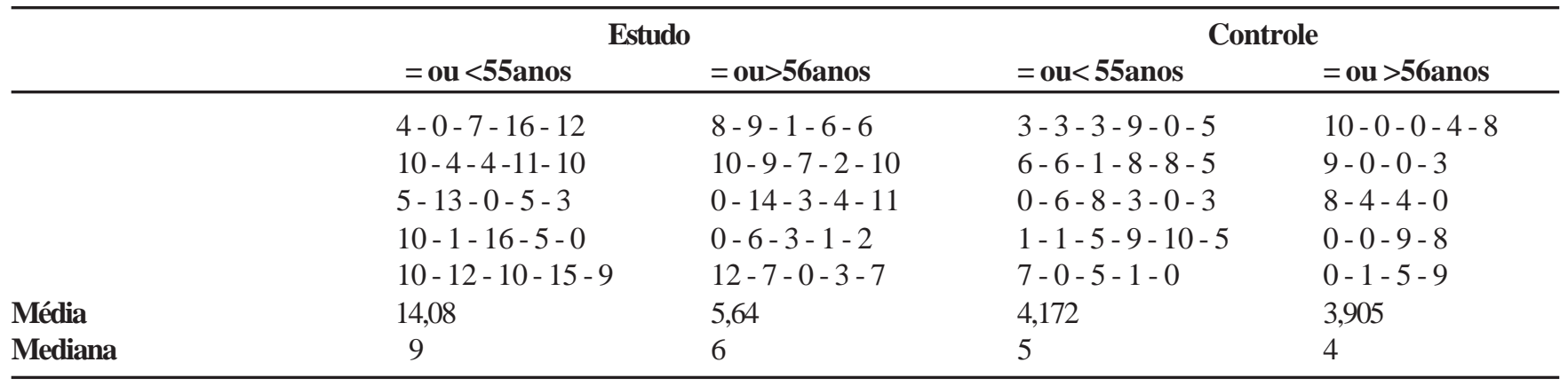

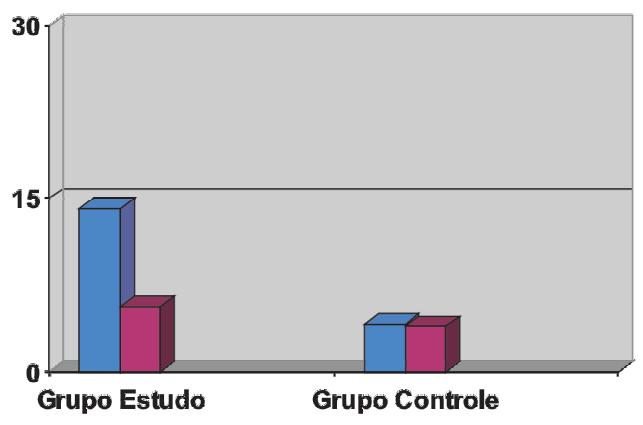

$\square<55$ anos $\square>56$ anos

Figura 1 - Faixas etárias do Grupo Estudo e do Grupo Controle segundo a Escala de Auto-estima Rosenberg UNIFESP-EPM e seus escores.

Tabela 4 - Tumores cutâneos conforme a localização na face e pescoço $(n=50)$.

\begin{tabular}{lll}
\hline Localização & N & \% \\
\hline Região Têmporo - frontal & 09 & $(18,0)$ \\
Região Zigomática & 01 & $(02,0)$ \\
Região orbital & 09 & $(18,0)$ \\
Região Oral & 09 & $(18,0)$ \\
Região Nasal & 19 & $(38,0)$ \\
Região da Bochecha & 02 & $(04,0)$ \\
Região Cervical & 01 & $(02,0)$ \\
\hline
\end{tabular}

Tabela 5 - Indivíduos com câncer de pele (grupo estudo) $e$ sem câncer de pele (grupo controle) segundo a classificação de Fitzpatrick.

\begin{tabular}{lcccc}
\hline Fototipos & Com câncer & Sem câncer & Total & \% \\
\hline I & 2 & 1 & 3 & 66,67 \\
II & 32 & 27 & 59 & 54,24 \\
III & 11 & 13 & 24 & 45,83 \\
IV & 5 & 3 & 8 & 62,50 \\
V + VI & 0 & 6 & 6 & 0,00 \\
n & 50 & 50 & 100 & 50,00 \\
\hline
\end{tabular}

Teste do Qui Quadrado

$X 2=7.424 \quad X 2=(4 g l ; 5 \%)=9.488$ aos raios ultravioleta (Tabela 5) $8,9,12-15$. O grupo controle foi representativo da população geral, selecionou-se aleatoriamente, de forma que uma comparação precisa pudesse ser feita entre a população geral e os pacientes com câncer de pele na face e no pescoço. A análise estatística mostrou homogeneidade quanto aos aspectos sócio-demográficos entre os dois grupos.

A exposição aos raios ultravioleta é um dos principais fatores de risco aos tumores cutâneos ${ }^{8,9,12,13}$, atingindo principalmente aqueles que se ocupam com atividades no campo, pessoas de pele clara, com manchas na pele, com olhos e cabelos claros. Também têm risco aumentado as pessoas que trabalham mais de três horas por dia ao ar livre, que se expõem e sofrem queimaduras freqüentes de sol e aqueles que possuem história familiar de câncer de pele 8,10,13,15. $^{\text {. }}$

A face e o pescoço são as regiões mais afetadas devido à maior exposição aos raios solares, sendo que os tumores nesta localização podem apresentar morbidade significati$\mathrm{va}^{12,15}$. Estes tumores podem levar a um grau variável de deformidade, com distúrbios funcional e cosmético, resultando em distúrbios psíquicos e sociais ${ }^{2}$.

Ainda, a percepção do estado de saúde e da qualidade de vida dos pacientes, bem como o impacto tanto de sua doença como de seu respectivo tratamento, estão sendo amplamente reconhecidos como um elemento importante de avaliação de resultados, sendo tópico de pesquisa em estudos clínicos e epidemiológicos ${ }^{16}$. Com o objetivo de avaliar individual e especificamente um aspecto da qualidade de vida dos pacientes com câncer de pele utilizou-se um instrumento validado, a Escala de Auto-estima de Rosenberg / UNIFESP-EPM 5. Tal procedimento proporciona uma detecção de melhora ou piora da auto-estima, objeto específico do presente estudo.

Escolheu-se a Escala de Rosenberg / UNIFESP-EPM como instrumento de medida de auto-estima por tratar-se de um questionário simples, bem como por ser um questionário disponível na literatura médica nacional. As suas principais vantagens são: capacidade de detectar pequenas alterações na auto-estima, possuir propriedades psicométricas, ser curto e de fácil entendimento ${ }^{17-19}$.

Outros estudos de qualidade de vida já publicados de pacientes portadores de carcinomas de pele, incluindo de cabeça e pescoço, não mostraram diminuição da qualidade de vida em relação à população geral ${ }^{2,3}$. 
No presente estudo, os pacientes portadores de carcinomas de pele da cabeça e do pescoço apresentaram menor auto-estima em relação ao grupo da população em geral, com medianas de 7,0 e 3,5, respectivamente, e com a hipótese calculada estatisticamente (Mann-Whitney) da amostra ser dife-

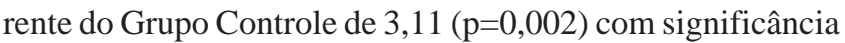
estatística (Tabela 3). Esses achados sugerem que o diagnóstico de câncer bem como a associação desta palavra com a letalidade e o lidar com a incerteza da doença, podem resultar em ansiedade e comprometimento de um dos aspectos da qualidade de vida que é a auto-estima ${ }^{20}$.

Neste estudo, a faixa etária dos pacientes com idade igual ou inferior a 55 anos foi a que mais apresentou os efeitos negativos sobre a auto-estima (Figura 1). Com perspectivas diferentes, pacientes mais idosos podem ter anseios que divergem dos mais jovens, se espiritualizando mais e dando menos importância a aspectos estéticos. Na literatura, encontrou-se que o grau de desconforto psicológico, quando relacionados ao impacto estético da cicatriz em pacientes com câncer de pele, estava associado às expectativas pré-cirúrgicas, podendo levar a um efeito negativo na auto-estima e, conseqüentemente, na qualidade de vida do indivíduo ${ }^{2,20}$.
A suposta influência de uma determinada localização do tumor na face, em resposta à Escala, não foi confirmada pela Análise de Variância de Kruskal Wallis ${ }^{7}(\mathrm{p}=0,322)$, que demonstrou não haver uma região mais importante que as outras. O mesmo acontecendo com a influência do tamanho do tumor nas respostas dos indivíduos do grupo estudo, que não se mostraram significantes nas suas variações, tornando nula a hipótese das amostras serem diferentes entre $\mathrm{si}(\mathrm{p}=0,126)$. Os tipos histológicos dos carcinomas cutâneos, podem se apresentar em formas variáveis, encontrando-se desde formas planas e não ulceradas, nodulares translúcidos, lesões eritematosas e descamativas com margens elevadas ${ }^{15}$, que pela longa evolução, em sua maioria, confundem o portador com lesões benignas e, às vezes, com episódios de involução e recrudescimento, que demoram a dar importância e até retardam a procura ao médico.

Os resultados deste trabalho demonstraram a existência de alterações na auto-estima dos indivíduos com carcinomas de pele da face e do pescoço, principalmente dos mais jovens, quando da percepção da doença e da necessidade do seu tratamento.

Os pacientes portadores de câncer de pele da face e do pescoço, exceto o melanoma, possuem menor auto-estima do que os pacientes sem câncer.

\begin{abstract}
Background: To evaluate self-esteem among patients with skin cancer of the face and the neck area. Methods: One hundred patients were studied, with ages ranging between 30 and 90 years old (median $=55$ years old). There were 44 males and 56 females. We divided them in two groups. One group had fifty patients with skin cancers, equal or larger than 10mm in the greater axis, and the pathological status was confirmed by previous biopsy. The control group had 50 patients without skin cancer. Rosenberg/UNIFESP-EPM's Self-esteem Scale was used to evaluate self-esteem. Data were analyzed by MannWhitney's test and Kruskal Wallis test. Results: There was no significant difference between the two groups in relation to age ( $p$ $=0.331$ ), sex and education. The mean tumor size was $20.9 \pm 2 \mathrm{~mm}$. Skin cancer patients presented significantly lower selfesteem, with average score of 6.84 and 3.90 for the control group $(p=0.002)$. Conclusion: Patients with skin cancer of the head and neck presented lower self-esteem than individuals without cancer.
\end{abstract}

Key words: Skin neoplasms; Carcinoma, basal cell; Carcinoma, squamous cell; head and neck neoplasms; Quality of life; Self concept.

\section{REFERÊNCIAS}

1. Miller DL, Weinstock MA. Nonmelanoma skin cancer in the United States: incidence. J Am Acad Dermatol. 1994;30(5 Pt1):774-8

2. Holfeld KI, Hogan DJ, Eldemire M, Lane PR. A psychosocial assessment of patients with basal cell carcinoma. J Dermatol Surg Oncol. 1990;16(8):750-3.

3. Rhee JS, Loberiza FR, Matthews BA, Neuburg M, Smith TL, Burzynski M. Quality of life assessment in nonmelanoma cervicofacial skin cancer. Laryngoscope. 2003;113(2):215-20.

4. Ciconelli RM, Ferraz MB, Santos W, Meinão I, Quaresma MR. Tradução para a língua portuguesa e validação do questionário genérico de avaliação de qualidade de vida SF-36 (Brasil SF-36). Rev Bras Reumatol. 1999;39(2):143-50.

5. Dini GM. Tradução para a língua portuguesa, adaptação cultural e validação do questionário de auto-estima de Rosenberg [Dissertação]. São Paulo: UNIFESP; 2000.

6. Fitzpatrick TB. The validity and practicality of sun-reactive skin types I through VI. Arch Dermatol. 1988;124(6):869-71.
7. Siegel S, Castellan Jr NJ. Nonparametric statistics. $2^{\text {nd }}$ edition. New York: McGraw-Hill; 1988.

8. Maia M, Proença NG, Moraes JC. [Risk factors for basal cell carcinoma: a case-control study]. Rev. Saúde Pública. 1995;29(1):27-37.

9. Santmyire BR, Feldman SR, Fleischer AB. Lifestyle high-risk behaviors and demographics may predict the level of participation in sun-protection behaviors and skin cancer primary prevention in the United States: results of the 1998 National health Interview Survey. Cancer. 2001;92(5):1315-24.

10. Lear JT, Tan BB, Smith AG, Bowers W, Jones PW, Heagerty AH, Strange RC, Fryer AA. Risk factors for basal cell carcinoma in the UK: case-control study in 806 patients. J R Soc Med. 1997;90(7):371-4.

11. Instituto Nacional do Câncer (INCa). Estimativa 2006: Incidência do câncer no Brasil. Disponível em: http://www.inca.gov.br/ vigilancia/

12. Marghoob AA. Basal cell and squamous cell carcinomas. What every primary care physician should know. Postgrad Med. 1997;102(2):139-42, 146, 152-4. 
13. Rhee JS, Matthews BA, Neuburg M, Smith TL, Burzynski M, Nattinger B. Quality of life and sun-protective behavior in patients with skin cancer. Arch Otolaryngol Head Neck Surg. 2004;130(2):141-6.

14. Hogan DJ, To T, Gran L, Wong D, Lane PR. Risk factors for basal cell carcinoma. Int J Dermatol. 1989;28(9):591-4.

15. Lear JD, Harvey I, Berker D, Strange RC, Fryer AA. Basal cell carcinoma. J R Soc Med. 1998;91(11):585-8.

16. Ferraz MB. Qualidade de vida. Conceito e um breve histórico. Jovem Médico. 1998; 4:219-20.

17. Jacobsen P, Holland JC. Psychological reactions to cancer surgery. In: Holland JC, Rowland JH (org). Handbook of psychooncology. New York: Oxford University Press; 1990. p. 117-33.

18. Faria FS, Guthrie E, Bradbury E, Brain AN. Psychosocial outcome and patient satisfaction following breast reduction surgery. Br J Plast Surg. 1999;52(6):448-52.

19. Ranzijn R, Keeves J, Luszcz M, Feather NT. The role of self perceived usefulness and competence in the self-esteem of elderly adults: confirmatory factor analyses of the Bachman revision of Rosenberg's Self-Esteem Scale. J Gerontol B Psychol Sci Soc Sci. 1998;53(2):P96-104.
20. Sarwer DB, Pertschuk MJ, Wadden TA, Whitaker LA. Psychological investigations in cosmetic surgery: a look back and a look ahead. Plast Reconstr Surg. 1998;101(4):113642 .

Como citar este artigo:

Carvalho MP, Oliveira Filho RS, Gomes HC, Veiga DF, Juliano Y, Ferreira LM. Auto-estima em pacientes com carcinomas de pele. Rev Col Bras Cir. [periódico na Internet] 2007; 34(6). Disponível em URL: http://www.scielo.br/rcbc

Endereço para correspondência:

Marcelo Prado de Carvalho

Rua Dr. Mário Gissoni, 25

Bairro João Paulo II

37550000 - Pouso Alegre - MG

Tel. (35) 9924-6752 Tel.Fax (35) 34492534

E-mail: mpczeta@yahoo.com.br 